\title{
Platelet Rich Fibrin as Regenerative Therapy for Peri Radicular Surgery in a Trauma Case - An Innovative Approach
}

\author{
Anshul Arora' ${ }^{1}$, Ashtha Arya ${ }^{2}$, Mandeep S. Grewal ${ }^{3}$, Shagun Patanjali ${ }^{4}$ \\ 1, 2, 3 Department of Conservative Dentistry and Endodontics, SGT University, Gurgaon, Haryana, India. \\ ${ }^{4}$ Department of Pedodontics \& Preventive Dentistry, SGT University, Gurgaon, Haryana.
}

\section{INTRODUCTION}

The success of endodontic therapy depends on complete repair and regeneration. Regenerative endodontics has added a future dimension in the current armamentarium of pulp therapeutics. Growth factors have contributed tremendously in regenerative molecular biology. PRF (Platelet-Rich Fibrin), a second-generation platelet concentrate has wide application in wound healing and alveolar bone repair. The present case report highlights the potential role of PRF for peri radicular surgery in a trauma case.

Dental trauma affects the tooth and supporting tissue and is often associated with pulpal necrosis with endodontic infection. Depending on the bacterial load and host immune response, endodontic infection progresses and flares into acute or chronic apical periodontitis. In cases where conventional root canal therapy fails to eliminate the lesion the periapical surgery is the treatment of choice. ${ }^{1}$ Periapical surgeries account for a substantial portion of endodontic practice and is aimed to remove periapical pathology and regeneration of lost bone and periodontium. ${ }^{2-3}$

Enhancement of regenerative process by using patient's own growth factors in blood is a powerful biological revolution in the field of dentistry. PRF is a second generation platelets concentrate with abundance of growth factor and are highly effective for inducing cell migration attachment and proliferation finally leading to regeneration. ${ }^{4} \mathrm{PRF}$ as an interpositional biomaterial release growth factor with its own biological scaffold accelerates and enhances the body's natural healing mechanisms. ${ }^{5}$ This case report presents the management of large periapical defect induced by trauma using PRF as a regenerative material.

\section{PRESENTATION OF CASE}

A 27-year-old patient reported to the Department of Conservative Dentistry and Endodontics with the complaint of pus discharge in relation to right maxillary anterior region. The patient had history of trauma due to an accident 6 years back. On clinical examination, there was grade I mobility in right central incisor and an associated draining sinus present on the buccal surface of 11 (Figure 1. Pre-operative clinical photograph).

Extra oral examination revealed no palpable lymph nodes. All vital signs were found to be within normal limits. Radiographic examination revealed a well-defined periapical radiolucency of about $1.2 \times 1.0 \mathrm{~cm}$ around the apices of maxillary right central and lateral incisors (Figure 2. Pre-operative radiograph). Clinically, the tooth was slightly hypersensitive to percussion and palpation. Pulp vitality test showed a negative response to temperature for both teeth. The diagnosis was pulpal necrosis with chronic apical abscess with respect to maxillary right central and lateral incisors.
Corresponding Author: Dr. Anshul Arora, Associate Professor, Faculty of Dental Sciences, SGT University, Gurgaon, Haryana, India. E-mail: anshularora2586@gmail.com

DOI: $10.14260 / j e m d s / 2020 / 808$

How to Cite This Article:

Arora A, Arya A, Grewal MS. et al. Platelet rich fibrin as regenerative therapy for periradicular surgery in a trauma case - an innovative approach. J Evolution Med Dent Sci 2020;9(48):3687-3690, DOI: $10.14260 /$ jemds $/ 2020 / 808$

Submission 14-07-2020,

Peer Review 15-10-2020,

Acceptance 21-10-2020,

Published 30-11-2020.

Copyright (c) 2020 Anshul Arora et al. This is an open access article distributed under Creative Commons Attribution License [Attribution 4.0 International (CC BY 4.0)] 


\section{DISCUSSION OF MANAGEMENT}

The treatment plan devised was root canal therapy in the maxillary right central and lateral incisors, but the lesion did not respond to the repeated calcium hydroxide dressings for 10 months. The dressing was changed at an interval of every 15 days as the canal was weeping at every subsequent visit. Later endodontic surgery was planned after taking an informed consent from the patient.

The canals were obturated with gutta-percha (Dentsply Maillefer, Ballaigues, Switzerland) and AH Plus sealer (Dentsply De Trey GmbH, Konstanz, Germany) under rubber dam isolation on the day of surgery. After effective local anaesthesia with $2 \%$ lignocaine, a full-thickness flap was reflected. A bony defect was visible on the buccal aspect of cortical plate with the lateral incisor, which was enlarged to aid in complete curettage of the granulation tissue (Figure 3. Full thickness flap reflection) and allowed room for retrograde instrumentation after root-end resection (Figure 4. Apicoectomy done). This was followed by irrigation with betadine (Win Medicare; Win Medicare Dental Products, New Delhi, India) and sterile saline solution. The root-end resection was carried out followed by retrograde cavity preparation with ultrasonic tips (Mectron S.P.A, Carasco, Italia). The retrograde cavity was filled with Mineral Trioxide Aggregate (MTA) (Pro Root MTA; Dentsply Maillefer). PRF preparation was done using the Choukroun's machine forming a strong fibrin matrix which remodelled slowly to natural blood clot. A blood sample was taken without anticoagulant in $10 \mathrm{ml}$ tubes, which was immediately centrifuged at $3000 \mathrm{rpm}$ for $10 \mathrm{~min}$ for collection, it was necessary to leave the PRF clots in a sterile metal cup for approximately $10 \mathrm{~min}$ to let slowly (Figure 5 . PRF) release the serum contained therein. The PRF clot was then packed into the defect to completely fill the bony crypt (Figure 6. Placement of PRF clot into the periapical defect). Wound closure was then obtained with 4 - 0 nylon sutures (Pearsalls Limited, Mumbai, India).

Nonsteroidal anti-inflammatory analgesics (Ibuprofen 500 $\mathrm{mg}$ ) were prescribed and the patient was advised to use chlorhexidine mouth wash for a week. The sutures were removed after 1 week, and satisfactory healing was observed. The patient was recalled at 3 and 12 months for revaluation. Follow-up radiographs at these intervals showed satisfactory bone regeneration in the periapical defect.
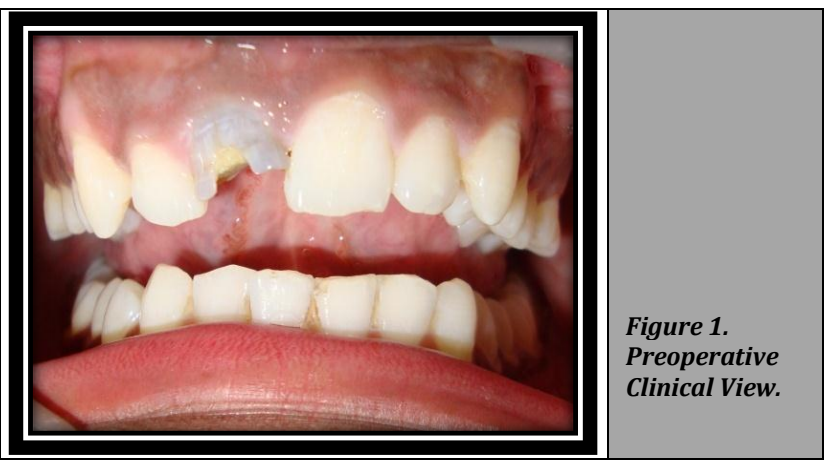
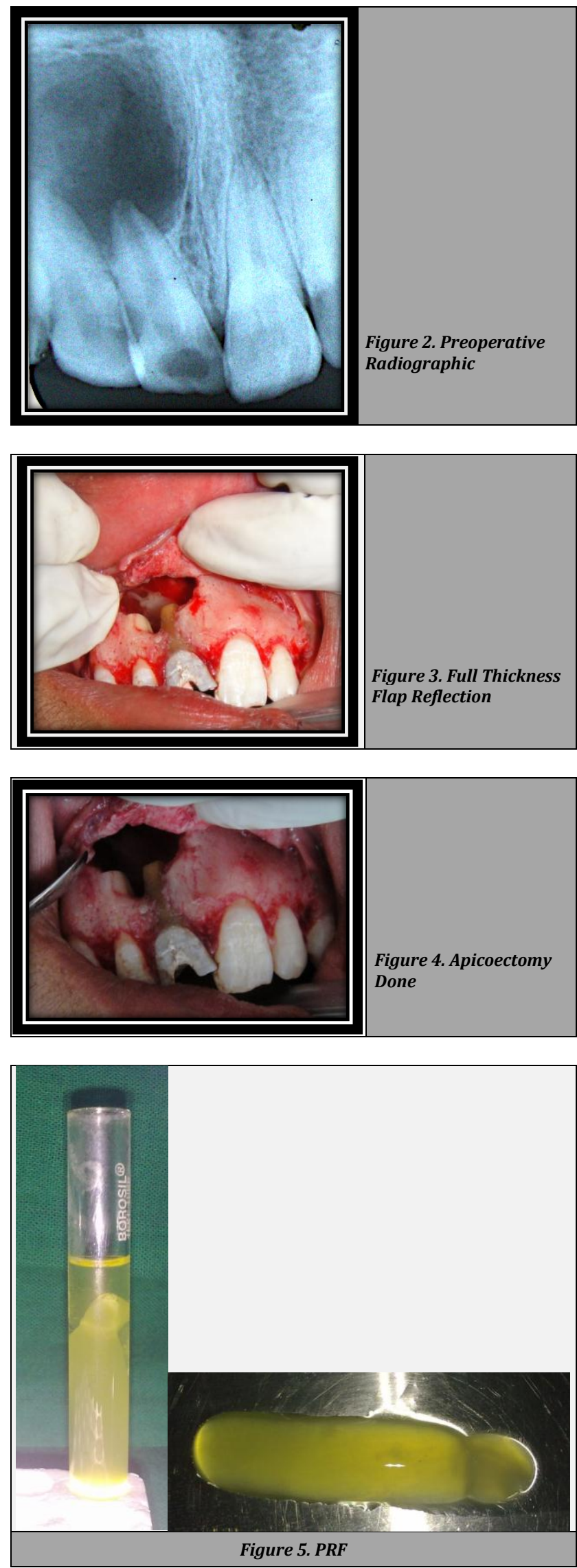

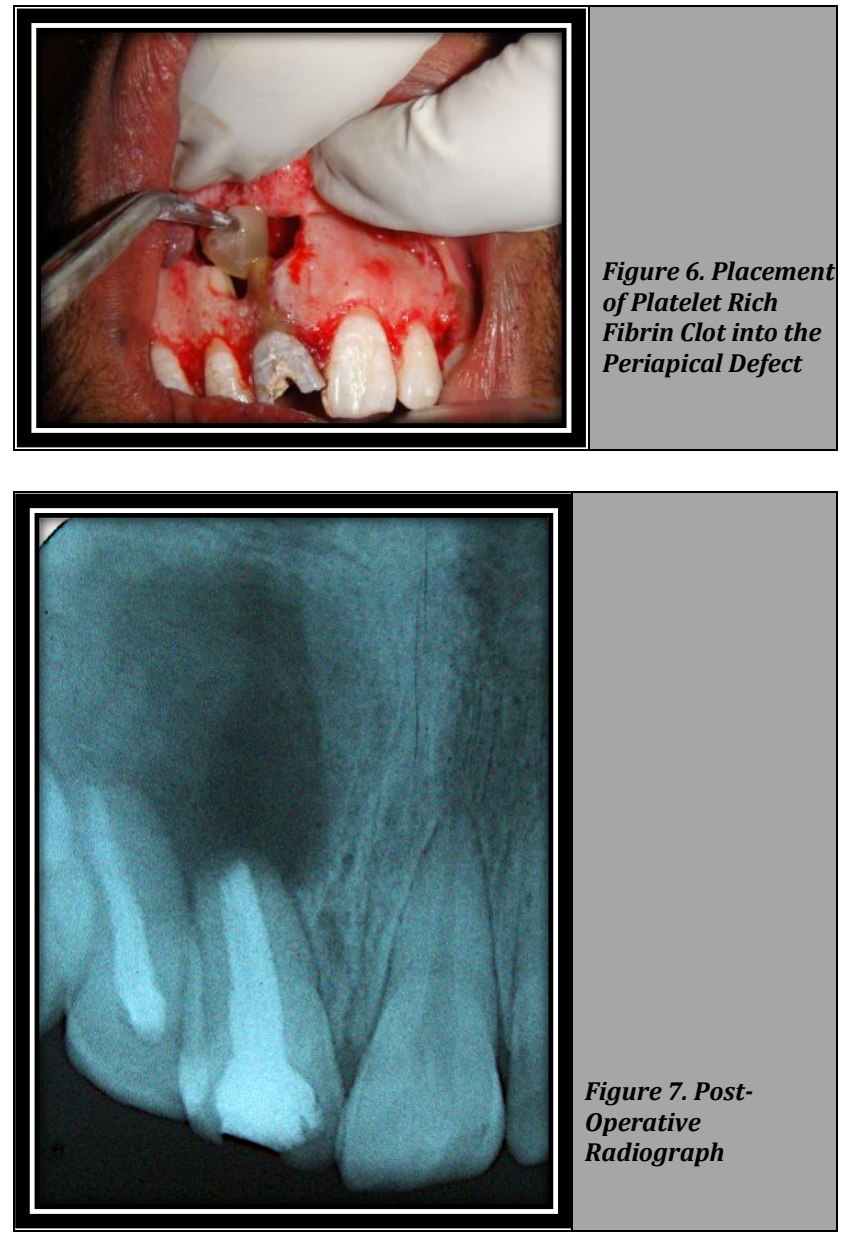

\section{DISCUSSION}

Endodontic surgery is often a necessary complement when conventional endodontic treatment has not yielded the desired healing outcome. Endodontic surgery is often undertaken due to the loss of bone tissue near the tooth, cortical bone and trabecular bone. After surgical procedure healing usually occurs by repair or regeneration. ${ }^{6}$

The present case report evaluates the clinical efficacy of PRF in the treatment of infrabony defect. PRF is the matrix of autologous fibrin with large quantity of growth factors embedded in it. Fibrin play an important role in wound repair by migration of fibroblast to provide extracellular matrix. Fibrin has also been reported to have a strong association angiogenesis and tissue remodelling.7-8 In response to the injury there is platelet activation as well as secretion of active protein for the formation of platelet plug. Growth factors such as Platelet-Derived Growth Factor (PDGF), transforming growth factor $\beta$ (TGF- $\beta$, including $\beta-1$ and $\beta$-2-isomers), Vascular Endothelial Growth Factor (VEGF), and Epidermal Growth Factor (EGF) can exert chemotactic effects towards the regenerative cells. Active protein secretion through these growth factor binds to trans membrane receptors of the target cells leading to collagen synthesis, wound healing and induction of new bone.

It has been reported that PRF clot forms a strong natural fibrin matrix and is an autologous biomaterial. Studies have demonstrated that PRF has shown no cytotoxicity toward many cells including preadipocytes, dermal prekeratinocytes, osteoblasts, oral epithelial cells, dental pulp cells, periodontal ligament cells, and gingival fibroblasts. With the above mentioned characteristics of platelets and advantages of the PRF in the present case reports, the periapical defects after the surgical procedure were filled with PRF to accelerate the physiologic healing mechanism. The postoperative clinical and radiographic assessments revealed an enhanced healing of the bony lesion at the 6 month follow-up.

Previously many case reports have been published using PRP / PRF as a graft material in periradiculardefects. ${ }^{9}$ According to Simonpierietal, ${ }^{10}$ the use of this platelet and immune concentrate during bone grafting offer additional advantages, thus causing rapid healing of periodontal tissue. Sunita et al ${ }^{11}$ stated that PRF made of intact platelets and fibrin yield an ideal scaffold for use in tissue repair.

\section{CONCLUSIONS}

PRF is a healing biomaterial as it contains all the factors required for optimal wound healing. Previous research and clinical experience seems to indicate that PRF improves early wound closure, maturation of bone and the final aesthetic result of periodontal soft tissues.

Financial or other competing interests: None.

Disclosure forms provided by the authors are available with the full text of this article at jemds.com.

\section{REFERENCES}

[1] Mazumbar P, Bhunia S. Treatment of periapical lesion with platelet rich fibrin. Indian Med Gazette 2013;4:2833.

[2] Singh S, Singh A, Singh S, et al. Application of PRF in surgical management of periapical lesions. Natl J Maxillofac Surg 2013;4(1):94-9.

[3] Mazumdar S, Joshi S, Ansari S. Experiences with the use of PRF (Plasma Rich Fibrin) in enucleated cystic cavity. J Indian Dent Assoc 2014;8(6):19-26.

[4] Su CY, Kuo YP, Tseng YH, et al. In vitro release of growth factors from platelet rich fibrin (PRF): a proposal to optimize the clinical applications of PRF. Oral Surg Oral Med Oral Pathol Oral Radiol Endod 2009;108(1):56-61.

[5] Ehrenfest DMD, de Peppo GM, Doglioli P, et al. Slow release of growth factors and thrombospondin-1 in Choukroun's platelet-rich fibrin (PRF): a gold standard to achieve for all surgical platelet concentrates technologies. Growth Factors 2009;27(1):63-9.

[6] Shivashankar VY, Johns DA, Vidyanath S, et al. Combination of platelet rich fibrin, hydroxyapatite and PRF membrane in the management of large inflammatory periapical lesion. J Conserv Dent 2013;16(3):261-4.

[7] Bhandari S, Ashwini TS, Naik R, et al. Regenerative periapical surgery: a case report. Dent Hypotheses 2013;4(2):61-6.

[8] Gassling V, Douglas T, Warnke PH, et al. Platelet-rich fibrin membranes as scaffolds for periosteal tissue engineering. Clin Oral Implants Res 2010;21(5):543-9. 
[9] Anantula K, Annareddy A. Platelet-rich fibrin (PRF) as an autologous biomaterial after an endodontic surgery. JNTR Univ Health Sci 2016;5(1):49-54.

[10] Simonpieri A, Del Corso M, Sammartino G, et al. The relevance of Choukroun's platelet-rich fibrin and metronidazole during complex maxillary rehabilitations using bone allograft. Part II: implant surgery, prosthodontics and survival. Implant Dent 2009;18(3):220-9.

[11] Singh S, Singh A, Singh S, et al. Application of PRF in surgical management of periapical lesions. Natl J Maxillofac Surg 2013;4(1):94-9. 